\title{
11. Glücklich bis ins hohe Alter? Lebenszufriedenheit und depressive Symptome in der zweiten Lebenshälfte
}

\author{
Julia K. Wolff \& Clemens Tesch-Römer
}

\section{Kernaussagen}

Im Jahr 2014 sind die meisten der 40- bis 85-Jährigen mit ihrem Leben zufrieden: Mehr als drei Viertel aller 40- bis 85-Jährigen geben an, dass sie mit ihrem Leben zufrieden sind - dieser Anteil ist in den höheren Altersgruppen sogar etwas größer. Der Anteil an Personen mit hoher Lebenszufriedenheit ist zwischen 1996 und 2002 um sechs Prozentpunkte angestiegen und von da an bis 2014 auf einem hohen Niveau (circa 78 Prozent) stabil geblieben.

Im höheren Alter sind mehr Menschen von zumindest leichten depressiven Symptomen betroffen als Jüngere: Menschen im höheren Alter berichten im Jahr 2014 häufiger von zumindest leichten depressiven Symptomen. Unter den 40- bis 54-Jährigen sind es 28,6 Prozent, bei den 55- bis 69-Jährigen 29,1 Prozent und unter den 70- bis 85-Jährigen ist dieser Anteil mit 33,1 Prozent etwas höher. Klinisch auffällige depressive Symptome sind dagegen mit circa sieben Prozent weniger weit verbreitet und unterscheiden sich nicht zwischen den Altersgruppen.

Der Wandel der depressiven Symptome unterscheidet sich zwischen den Altersgruppen - nur Ältere berichten 2014 weniger Symptome als 2002: Unter den 66- bis 83-Jährigen haben im Jahr 2014 weniger Menschen zumindest leichte depressive Symptome als noch 2002. Bei den Jüngeren (42 bis 65 Jahre) ist dieser Anteil nur zwischen 2002 und 2008 kleiner geworden, von 2008 zu 2014 hat er jedoch wieder zugenommen.

Mehr Frauen als Männer berichten von zumindest leichten depressiven Symptomen: Frauen leiden im Jahr 2014 häufiger unter zumindest leichten depressiven Symptomen als Männer (33,6 Prozent versus 25,9 Prozent). Dies gilt insbesondere für die Altersgruppe der 70- bis 85-Jährigen. In dieser Altersgruppe sind Frauen zudem seltener zufrieden (76,7 Prozent) mit ihrem Leben als Männer (84,3 Prozent).

Niedriggebildete haben häufiger eine geringere Lebenszufriedenheit und häufiger zumindest leichte depressive Symptome als Höhergebildete: Bildungsunterschiede in der Lebenszufriedenheit und der depressiven Symptomatik zeigen sich in allen Altersgruppen der zweiten Lebenshälfte. Die Bildungsungleichheit in der depressiven Symptomatik hat sich zwischen 2002 und 2014 nicht verändert. Bildungsunterschiede bei der Lebenszufriedenheit hatten zwischen den Jahren 1996 und 2002 zunächst zugenommen, sind von 2002 zu 2014 aber stabil geblieben. 


\subsection{Einleitung}

Ein gutes Leben im Alter wird durch viele Faktoren, wie Gesundheit, materielle Lage oder Wohnsituation bestimmt. Ein wichtiger Bestandteil eines guten Lebens ist auch das subjektive Wohlbefinden, also wie es einer Person geht und wie sie sich fühlt. In der Alternsforschung wird unter anderem die Zufriedenheit mit dem eigenen Leben als ein Indikator für ein hohes subjektives Wohlbefinden angesehen. Die allgemeine Lebenszufriedenheit betrifft die Lebenssituation einer Person im Allgemeinen: Wer im Alter eine hohe Lebenszufriedenheit äußert, der - so die Annahme - lebt ein gutes Leben im Alter.

In vielen Untersuchungen hat sich gezeigt, dass die Lebenszufriedenheit im Verlauf des Älterwerdens in der Regel sehr stabil ist, obwohl man annehmen könnte, dass (chronische) Erkrankungen, Beeinträchtigungen der körperlichen Beweglichkeit oder der Verlust von Partnerin beziehungsweise Partner oder befreundeten Personen die Zufriedenheit mit dem eigenen Leben einschränken müssten (Staudinger 2000). Dieser Befund - hohe Zufriedenheit im Alter trotz vieler Verlustereignisse - ist als ,Paradox der Lebenszufriedenheit im Alter' bezeichnet worden. Erst im sehr hohen Alter und insbesondere in der Phase vor dem Lebensende nimmt die Lebenszufriedenheit ab (Gerstorf, Ram, Estabrook, Schupp, Wagner, \& Lindenberger 2008), sodass man argumentieren könnte, dass weniger das Alter als vielmehr der zeitliche Abstand zum Lebensende bedeutsam für die Lebenszufriedenheit einer Person ist. Als Erklärung für die hohe Stabilität der Lebenszufriedenheit im Alter wird häufig auf sich verändernde Vergleichsstandards verwiesen: Bei der Beurteilung der Zufriedenheit verändern sich mit dem Alter bei den meisten Menschen die Kriterien dafür, was sie als zufriedenstellend bezeichnen. Durch die Anpassung dieser Kriterien bleibt die Zufriedenheit mit dem eigenen Leben auch angesichts von gesundheitlichen oder sozialen Verlusten weiterhin hoch. Auch mit Blick auf Frauen und Männer ist zu konstatieren: Geschlechtsunterschiede bei der Lebenszufriedenheit sind meist klein oder nicht zu finden (Tesch-Römer, MotelKlingebiel, \& Tomasik 2008).

Allerdings finden sich durchaus Unterschiede in der Lebenszufriedenheit zwischen anderen gesellschaftlichen Gruppen. Am bedeutsamsten sind hierbei Unterschiede zwischen Bildungsgruppen: Aus der Literatur ist bekannt, dass Höhergebildete eine höhere Lebenszufriedenheit als niedriger Gebildete berichten (Laubach, Schumacher, Mundt, \& Brähler 2000; Pinquart \& Sörensen 2000). Bildungsunterschiede sind auch ein Indikator für sozioökonomische Unterschiede. Höhergebildete Personen haben neben besseren Kenntnissen und Kompetenzen häufig auch ein höheres Einkommen und berufliches Ansehen und damit mehr Ressourcen für eine hohe Lebenszufriedenheit als Personen mit niedrigerem Bildungsniveau. Zudem gibt es in Deutschland auch regionale Unterschiede bei der Lebenszufriedenheit. Auch ein Vierteljahrhundert nach der Wiedervereinigung sind Unterschiede zwischen Ost- und Westdeutschland in der Lebenszufriedenheit zu finden (Schupp, Goebel, Kroh, \& Wagner 2013).

Bei der bisherigen Argumentation ist $\mathrm{zu}$ berücksichtigen, dass die Lebenszufriedenheit nur einer von mehreren Indikatoren für das subjektive Wohlbefinden im Alter ist. Lebenszufriedenheit ist das Resultat von individuellen Überlegungen und Vergleichen: Die eigene Lebenssituation wird mit der von der Person angestrebten Lebenssituation verglichen. Dies ist eine kognitive Facette des subjektiven Wohlbefindens. Davon zu unterscheiden ist die emotionale Facette des subjektiven Wohlbefindens. Dabei geht es um den Gefühlszustand einer Person, also um die Frage, ob sich ein Mensch glücklich oder niedergeschlagen, fröhlich oder traurig, motiviert oder antriebslos fühlt. In diesem Kapitel untersuchen wir in diesem Zusammenhang depressive Symptome, also Gefühle der Niedergeschlagenheit, der Antriebslosigkeit und der Verstimmung.

Auch wenn emotionale und kognitive Komponenten des subjektiven Wohlbefindens miteinander verknüpft sind (in der Regel zeigen Men- 
schen, die mit ihrem Leben zufrieden sind, eine nur geringe depressive Symptomatik), sind doch Unterschiede zu beobachten. Gefühle sind im Vergleich mit Zufriedenheitsurteilen weniger stark vermittelt und geprüft - sie bilden Unterschiede im Wohlbefinden und die Reaktion auf Veränderungen der Lebenssituation möglicherweise besser $\mathrm{ab}$ als eine rationale Bewertung.

Dies zeigt sich insbesondere bei Altersunterschieden. Während bei der Lebenszufriedenheit bis ins höhere Erwachsenenalter nur geringe Altersunterschiede feststellbar sind, stellt sich dies mit Blick auf depressive Symptome anders dar. Im hohen Alter zeigen sich insbesondere bei Menschen mit erheblichen gesundheitlichen Einschränkungen verstärkt depressive Symptome (Chang-Quan, Bi-Rong, Zhen-Chan, Ji-Rong, \& Qing-Xiu 2010; Rothermund \& Brandtstädter 2003).

Zudem sind im Gegensatz zur Lebenszufriedenheit deutliche Geschlechtsunterschiede bei der depressiven Symptomatik zu finden: Frauen äußern in der Regel mehr und stärkere depressive Symptome als Männer (Buber \& Engelhardt 2011; Tesch-Römer et al. 2008). Dies kann einerseits daran liegen, dass Frauen häufiger über vor allem negative Gefühle berichten (vgl. Schienle 2007). Andererseits können sich darin auch geschlechtsspezifische Ungleichheiten hinsichtlich der Ressourcen, zum Beispiel Einkommen oder berufliche Aufstiegschancen, widerspiegeln. Auch Belastungen, von denen Frauen stärker betroffen sind als Männer, wie die Vereinbarung von Erwerbstätigkeit und Unterstützung von gesundheitlich eingeschränkten Personen oder Kinderbetreuung, können sich in vermehrten negativen Gefühlen ausdrücken (vgl. Kapitel 12 und 15). Ähnlich zur Lebenszufriedenheit zeigt sich ein deutlicher Bildungsgradient in der Häufigkeit von depressiven Symptomen (Bjelland, Krokstad, Mykletun, Dahl, Tell, \& Tambs 2008; Fryers, Melzer, \& Jenkins 2003).

Mit Blick auf regionale Unterschiede in Deutschland zeigt sich in der Literatur ein gemischtes Bild: Entgegen der Erwartungen sind depressive Erkrankungen in Westdeutschland häufiger als in Ostdeutschland (Müters, Hoebel, \& Lange 2013). Allerdings belegen Studien, die eher die psychische Befindlichkeit beziehungs- weise negative Emotionen und nicht manifeste Diagnosen untersuchen, höhere oder gleich hohe psychische Beschwerden in Ostdeutschland im Vergleich zu Westdeutschland (Albani, Gunzelmann, Schmutzer, Grulke, Bailer, Blaser, Greyer, \& Brähler 2005). Zudem ist mit Blick auf die zweite Lebenshälfte die Frage zu stellen, ob sich Unterschiede zwischen Frauen und Männern, zwischen Bildungsgruppen und zwischen Ost- und Westdeutschland in allen Altersgruppen in ähnlicher Weise zeigen oder möglicherweise mit dem Alter stärker (oder schwächer) werden (vgl. Altersunterschiede in Bildungseffekten; Schöllgen, Huxhold, \& Tesch-Römer 2010).

Eine zentrale Aufgabe des Deutschen Alterssurveys (DEAS) ist es auch, der Frage nachzugehen, ob sich die Lebenssituationen von älteren Menschen seit 1996 geändert haben. Mit Blick auf Lebenszufriedenheit und depressive Symptome kann man also fragen, ob Menschen in der zweiten Lebenshälfte zufriedener (oder unzufriedener) sowie weniger niedergeschlagen (oder niedergeschlagener) geworden sind. Für die Lebenszufriedenheit zeigt sich in den Daten des Sozio-oekonomischen Panels (SOEP) bis 2013 ein positiver Wandel (Schupp et al. 2013) - allerdings differenziert für Ost- und Westdeutschland. Laut Daten des SOEP bestehen auch 2013 trotz einer Annäherung an Westdeutschland noch Unterschiede bei der Lebenszufriedenheit zwischen den beiden Landesteilen (Priem \& Schupp 2014). Wenn man mit Veenhoven (2008) argumentiert, dass die Lebenszufriedenheit zeigt, wie gut die Gesellschaft die Bedürfnisse ihrer Bürger befriedigt, könnte die mittlerweile ein Vierteljahrhundert zurückliegende Wiedervereinigung Deutschlands als natürliches Experiment angesehen werden. Es gilt demnach zu untersuchen, ob es auch in der zweiten Lebenshälfte zu einer weiteren Angleichung zwischen Ost- und Westdeutschland bezüglich der Lebenszufriedenheit gekommen ist.

Für den Wandel der depressiven Symptome zeigt sich möglicherweise ein anderes Bild. Laut Ergebnissen der Panelstudie belgischer Haushalte, die Personen bis 75 Jahren umfasst, ist die depressive Symptomatik im Zeitraum von 1992 bis 2002 in jüngeren Kohorten höher als in äl- 
teren Kohorten (Brault, Meuleman, \& Bracke 2012). Diese Zunahme wird begründet mit einer steigenden Pathologisierung von negativen Emotionen. In repräsentativen amerikanischen und kanadischen Stichproben wurden ähnliche Befunde zu einer steigenden negativen Befindlichkeit in der zweiten Lebenshälfte über die Kohorten gefunden (Keyes, Nicholson, Kinley, Raposo, Stein, Goldner, \& Sareen 2014). Dies spiegelt sich auch in einem Anstieg von Depressionsprävalenzen von 2009 zu 2012 in Deutschland wider (Robert Koch-Institut 2014). Es ist eine offene Frage, ob sich dieser Wandel der depressiven Symptomatik auch im DEAS zeigt und ob er alle Altersgruppen gleichermaßen betrifft.

Angesichts der großen Bildungsunterschiede bei der Lebenszufriedenheit und der depressiven Symptomatik, stellt sich zudem die Frage, ob sich diese sozialen Ungleichheiten seit 1996 verändert haben. Zwischen 1996 und 2008 ha- ben im DEAS die Bildungsunterschiede bei der Lebenszufriedenheit über die Kohorten zugenommen (Tesch-Römer, Wiest, \& Wurm 2010). Daher soll in diesem Kapitel auch der Frage nachgegangen werden, ob sich die Bildungsunterschiede seit 1996 in der Lebenszufriedenheit und seit 2002 in den depressiven Symptomen (weiter) verändert haben.

Zusammenfassend werden im vorliegenden Kapitel zwei Themenkomplexe untersucht:

1. Welche Altersunterschiede zeigen sich im Jahr 2014 hinsichtlich Lebenszufriedenheit und depressiver Symptome? Bestehen zusätzlich oder zudem auch Unterschiede zwischen anderen gesellschaftlichen Gruppen?

2. Wie haben sich die Lebenszufriedenheit (1996 bis 2014) und die depressiven Symptome (2002 bis 2014) gewandelt?

\subsection{Datengrundlage ${ }^{1}$}

Daten. Zur Beantwortung der Fragestellungen werden die Daten der 40- bis 85-Jährigen in den Jahren 1996, 2002, 2008 und 2014 genutzt. Für die Lebenszufriedenheit, die im schriftlichen Fragebogen erfasst wird, liegen Daten zu allen vier Zeitpunkten vor. Die depressiven Symptome werden seit 2002 im mündlichen Interview erhoben.

Lebenszufriedenheit. Die Lebenszufriedenheit wurde mit fünf Aussagen erfasst (Glaesmer, Grande, Braehler, \& Roth 2011; Pavot \& Diener 1993), wie etwa „In den meisten Dingen ist mein Leben ideal.“, „Ich bin zufrieden mit meinem Leben.“ und „Wenn ich mein Leben noch einmal leben könnte, würde ich kaum etwas anders machen.“. Den Befragten wurde eine fünfstufige Skala vorgelegt mit den Abstufungen, trifft genau $\mathrm{zu}^{\prime}(5)$, ,trifft eher zu' (4), ,weder noch (3), ,trifft eher nicht zu' (2), ,trifft gar nicht zu' (1). Anhand dieser Antwortmöglichkeiten schätzten die Be-

1 Die Daten des DEAS können für wissenschaftliche Zwecke kostenlos beim Forschungsdatenzentrum des DZA (www.fdz-dza.de) bezogen werden. fragten ein, inwiefern die Aussagen auf sie selbst zutreffen. Aus den Antworten auf alle fünf Fragen wurde ein Mittelwert gebildet. Wenn eine Person einen Mittelwert größer als 3,3 aufweist, bedeutet dies, dass die Person im Durchschnitt den Aussagen (eher) zugestimmt hat. Berichtet wird der Anteil der Personen, die nach dieser Definition eine hohe Lebenszufriedenheit aufweisen.

Depressive Symptome. Die depressiven Symptome wurden mit der 15 Items umfassenden Kurzfassung der Allgemeinen Depressionsskala gemessen (ADS-K; Hautzinger \& Bailer 1993). Die ADS-K ist ein Selbstbeurteilungsinstrument, mit dem emotionale, motivationale, kognitive, somatische und interaktionale Beschwerden erfragt werden, zum Beispiel „In der letzten Woche war ich deprimiert/niedergeschlagen.“, „In der letzten Woche war alles anstrengend für mich.", „In der letzten Woche hatte ich das Gefühl, dass mich Leute nicht leiden können.“. Den Befragten wurde eine vierstufige Skala vorgelegt mit den Abstufungen, selten/überhaupt nicht; weniger als ein Tag lang' (0), ,manchmal; ein bis zwei Tage 
lang' (1), ,öfters; drei bis vier Tage lang' (2) und ,meistens, die ganze Zeit; fünf bis sieben Tage lang' (3). Um Gruppenunterschiede und Veränderungen im Wandel darzustellen, wird in diesem Kapitel ein ADS-K-Summenwert größer als acht verwendet. Eine Person, die mehr als acht Punkte im ADS-K aufweist, gibt entweder für mehr als die Hälfte der Items an, unter den Symptomen mindestens ,manchmal; ein bis zwei Tage lang zu leiden oder ist zwar nur von einzelnen Symptomen, aber dafür häufiger im Alltag betroffen. Berichtet wird demnach der Anteil an Personen, die mindestens leichte depressive Symptome aufweisen. Bei Untersuchungen größerer Bevölkerungsgruppen wie dem DEAS weist ein ADS-K-Summenwert von größer als 18 Punkten auf die Möglichkeit einer ernsthaften depressiven Störung hin. Daher wird zusätzlich der Anteil an Personen mit klinisch auffälligen depressiven Symptomen für das Jahr 2014 berichtet.

Gruppierungsvariablen. Als Gruppierungsvariablen werden im vorliegenden Kapitel die drei großen Altersgruppen, welche die Stratifizierung der Stichprobe widerspiegeln (40bis 54-Jährige, 55- bis 69-Jährige und 70- bis 85-Jährige), die drei Bildungsgruppen (niedrige, mittlere und hohe Bildung) basierend auf der re- duzierten ISCED-Klassifikation, das Geschlecht der Befragten und die Regionseinteilung Ostdeutschland versus Westdeutschland verwendet (vgl. Kapitel 2). Bei den Fragestellungen zum Wandel in der Lebenszufriedenheit und in den depressiven Symptomen, die im DEAS seit 1996 (beziehungsweise 2002) alle sechs Jahre wiederholt erfragt werden, ist es sinnvoll, Altersunterschiede im Wandel anhand von Sechs-JahresAltersgruppen zu untersuchen. Diese Aufteilung ermöglicht es, über die Erhebungszeitpunkte hinweg Personen gleichen Alters miteinander zu vergleichen, die aus nicht überlappenden $\mathrm{Ge}$ burtsjahrgängen stammen ( 42 bis 47 Jahre, 48 bis 53 Jahre, 54 bis 59 Jahre, 60 bis 65 Jahre, 66 bis 71 Jahre, 72 bis 77 Jahre, 78 bis 83 Jahre).

Analysen. In den Abbildungen und im Text sind gewichtete (gruppenspezifische) prozentuale Verteilungen angegeben. Um Gruppenunterschiede auf Signifikanz zu testen, wurden logistische Regressionen unter Kontrolle der Stratifizierungsvariablen Altersgruppe (40bis 54-Jährige, 55- bis 69-Jährige und 70- bis 85-Jährige), Geschlecht und Region (West-/Ostdeutschland) berechnet. Das genaue Vorgehen ist in Kapitel 2 beschrieben.

\subsection{Lebenszufriedenheit und depressive Symptome im Jahr 2014}

Abbildung 11-1 stellt den Anteil an Personen mit hoher Lebenszufriedenheit, zumindest leichten depressiven Symptomen und klinisch auffälligen depressiven Symptomen nach den drei Altersgruppen dar.

\section{Im Jahr 2014 sind die meisten der 40- bis 85-Jährigen mit ihrem Leben zufrieden.}

Mehr als drei Viertel der Menschen zwischen 40 und 85 Jahren geben an, mit dem Leben eher oder sehr zufrieden zu sein (77,8 Prozent). Da- bei gibt es, wie erwartet, geringe Altersunterschiede. Die 70 - bis 85 -Jährigen sind zu einem etwas größeren Prozentsatz zufrieden mit ihrem Leben als die beiden jüngeren Altersgruppen (Abbildung 11-1).

Eine hohe Lebenszufriedenheit schützt offensichtlich nicht alle Personen davor, zumindest bisweilen niedergeschlagen, traurig oder lustlos zu sein. Der Anteil der Personen mit mindestens leichten depressiven Symptomen, liegt in allen Altersgruppen um die 30 Prozent (Abbildung 11-1). 


\section{Im höheren Alter sind mehr Menschen von zumindest leichten depressiven Symptomen betroffen als Jüngere.}

Trotz einer höheren Lebenszufriedenheit unter den 70- bis 85-Jährigen berichtet diese Altersgruppe auch häufiger zumindest leichte depressive Symptome. Der Anteil von Personen mit klinisch auffälliger depressiver Symptomatik ist dagegen über die drei Altersgruppen sta- bil. Frauen berichten häufiger als Männer (9,8 Prozent versus 5,1 Prozent) klinisch auffällige depressive Symptome (vgl. Tabelle A 11-3 im Anhang). Diese Werte liegen in etwa bei den Prozentsätzen, die auch das Robert Koch-Institut berichtet, wonach zehn Prozent der Frauen und sechs Prozent der Männer angaben, im letzten Jahr von einer Ärztin oder einem Arzt eine Depression diagnostiziert bekommen zu haben (Busch, Maske, Ryl, Schlack, \& Hapke 2013).

Abbildung 11-1: Anteile der Personen mit hoher Lebenszufriedenheit, mindestens leichten depressiven Symptomen und klinisch auffälligen depressiven Symptomen, nach Alter, im Jahr 2014 (in Prozent)

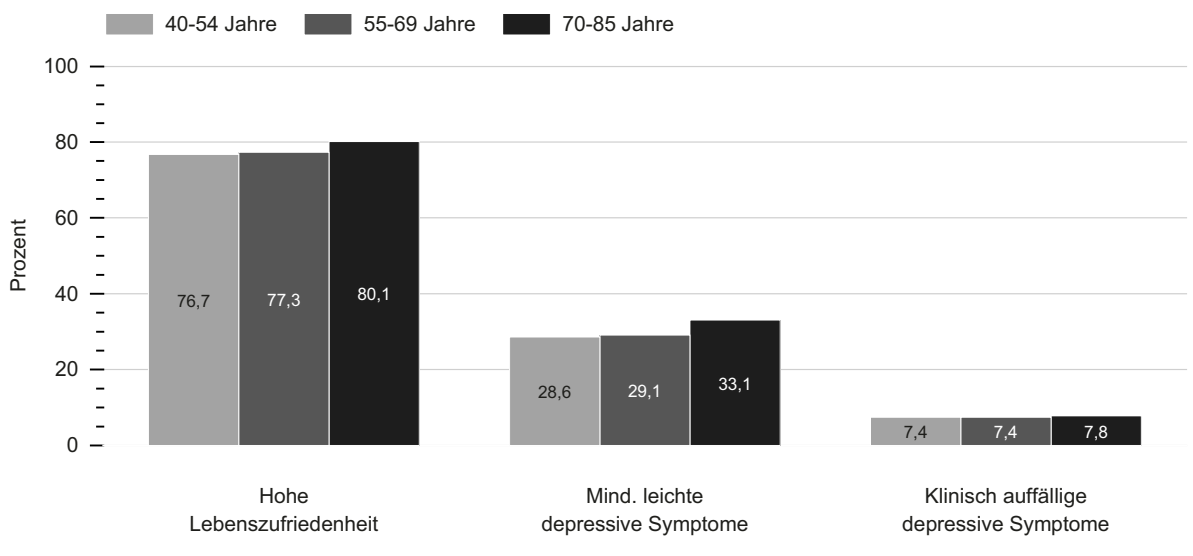

Quelle: DEAS 2014 ( $n=4.258$ für Lebenszufriedenheit und $n=5.858$ für depressive Symptome), gewichtet, gerundete Angaben; $(p<, 05)$.

Die 70- bis 85-Jährigen unterscheiden sich signifikant von den anderen Altersgruppen für Lebenszufriedenheit und für leichte depressive Symptome. Keine signifikanten Altersgruppenunterschiede für klinisch auffällige depressive Symptome.

Die Unterschiede bei der Lebenszufriedenheit zwischen Männern und Frauen sind, wie erwartet, gering (Abbildung 11-2a). In der Gesamtstichprobe unterscheiden sich Männer und Frauen statistisch nicht bedeutsam. Betrachtet man die drei Altersgruppen separat, so zeigt sich nur für die älteste Altersgruppe (70- bis 85-Jährige) ein anderes Bild: In diesem Alter geben etwas weniger Frauen eine hohe Lebenszufriedenheit an als Männer (76,7 Prozent versus 84,3 Prozent; vgl. Tabelle A 11-1 im Anhang).

\section{Mehr Frauen als Männer berichten von zumindest leichten depressiven Symptomen.}

Deutlicher ist der Geschlechtsunterschied beim Anteil an Menschen mit zumindest leichten depressiven Symptomen. Frauen sind insgesamt häufiger von depressiven Symptomen betroffen (Abbildung 11-2b). Dieser Unterschied findet sich in allen drei Altersgruppen und ist in höheren Altersgruppen sogar größer (vgl. Tabelle A 11-2 im Anhang). Bei den 40- bis 54-Jährigen beträgt der Geschlechtsunterschied 5,1 Prozentpunkte, bei den 55- bis 69-Jährigen 7,3 Prozentpunkte und bei den 70 - bis 85 -Jährigen 12,2 Prozentpunkte. Wir sehen also vor allem in der depressiven Symptomatik und bei der ältesten Altersgruppe, dass Frauen ein schlechteres Wohlbefinden berichten als Männer. 
Abbildung 11-2: Anteile der Personen mit hoher Lebenszufriedenheit und mindestens leichten depressiven Symptomen, gesamt, nach Geschlecht, Bildung und Landesteil, im Jahr 2014 (in Prozent)

a) Anteile der Personen mit hoher Lebenszufriedenheit

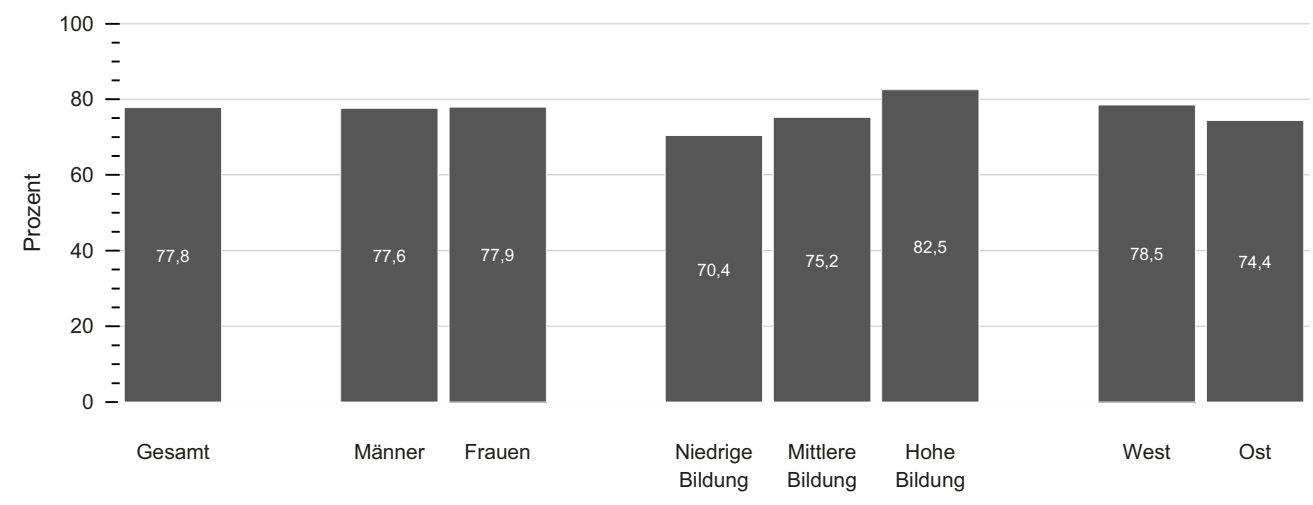

b) Anteile der Personen mit mindestens leichten depressiven Symptomen

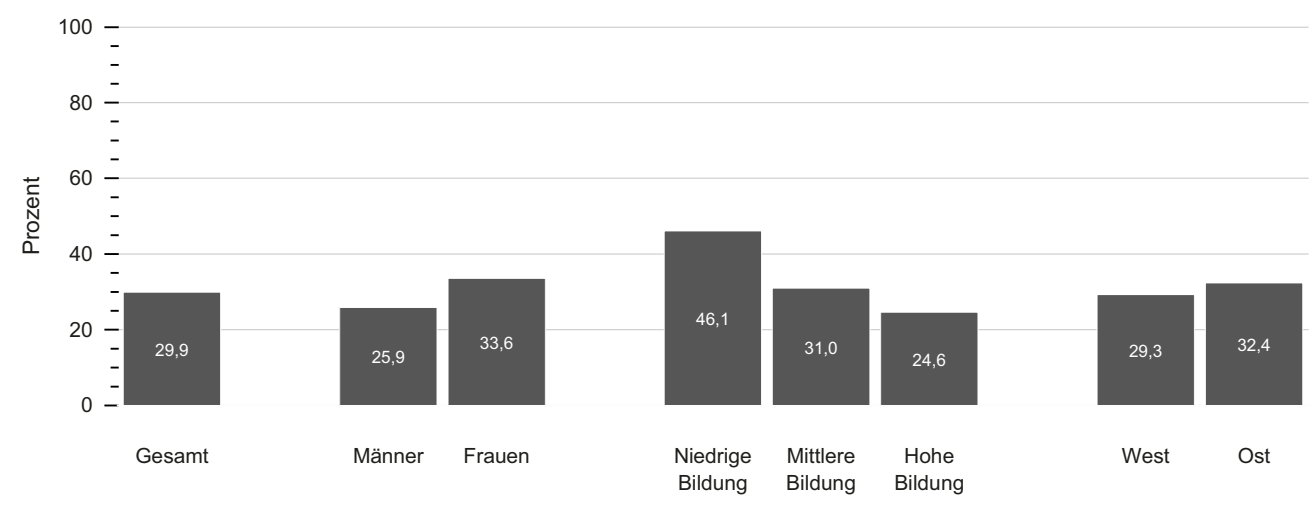

Quelle: DEAS 2014 ( $n=4.258$ für Lebenszufriedenheit und $n=5.858$ für depressive Symptome), gewichtet, gerundete Angaben; $(p<, 05)$.

a) Keine signifikanten Geschlechtsunterschiede. Signifikante Bildungsgruppen- und Regionsunterschiede. b) Signifikante Unterschiede nach Geschlecht, Bildungsgruppe und Region.

\section{Niedriggebildete haben häufiger eine geringere Lebenszufriedenheit und häufiger zumindest leichte depressive Symptome als Höhergebildete.}

Sehr deutliche Unterschiede sowohl in der Lebenszufriedenheit als auch in depressiven Symptomen sind zwischen den Bildungsgruppen $\mathrm{zu}$ erkennen (Abbildung 11-2a und 11-2b). In der Gruppe der Personen mit niedriger Bildung ist der Anteil der Personen mit hoher Lebenszufriedenheit geringer (70,4 Prozent) und der Anteil der Personen mit zumindest leichten depressiven Symptomen deutlich höher (46,1 Prozent) als in der Gruppe der Personen mit mittlerer (75,2 Prozent und 31,0 Prozent) und höherer Bildung (82,5 Prozent und 24,6 Prozent). Aber auch die beiden Gruppen der Personen mit mittlerer und höherer Bildung unterscheiden sich. Diese deutlichen Bildungsunterschiede finden sich in allen Altersgruppen gleichermaßen. 
Auch 25 Jahre nach der Wende berichten Ostdeutsche seltener eine hohe Zufriedenheit und häufiger depressive Symptome als Westdeutsche.

Auch im Jahr 2014 finden sich regionale Unterschiede zwischen Ost- und Westdeutschland in der Lebenszufriedenheit und den depressiven Symptomen (Abbildung 11-2a und 11-2b). Der
Anteil von zufriedenen Personen ist in Westdeutschland um circa vier Prozentpunkte höher als in Ostdeutschland. Zugleich liegt der Anteil von Personen mit zumindest geringen depressiven Symptomen in Ostdeutschland um circa drei Prozentpunkte über dem Anteil dieser Personengruppe in Westdeutschland. Diese regionalen Unterschiede finden sich in allen Altersgruppen gleichermaßen.

\subsection{Lebenszufriedenheit und depressive Symptome in den Jahren 1996, 2002, 2008 und 2014}

Wie hat sich die Lebenszufriedenheit in der zweiten Lebenshälfte seit 1996 gewandelt? Sind Menschen im Jahr 2014 in der zweiten Lebenshälfte zufriedener (oder unzufriedener) mit ihrem Leben? Und wie sieht es mit dem Auftreten depressiver Symptome aus? Hat der Anteil von Menschen, die über depressive Symptome berichten, seit 2002 zugenommen (oder abgenommen)?

\section{Der Anteil an Personen mit hoher}

Lebenszufriedenheit ist von 1996 zu 2002 angestiegen und bleibt seitdem stabil hoch.
Ein Blick auf die Befunde verrät eine hohe Stabilität des subjektiven Wohlbefindens in der zweiten Lebenshälfte. In allen Jahren hat der Anteil der Menschen, die angeben mit ihrem Leben eher oder sehr zufrieden zu sein, bei über 70 Prozent gelegen (Abbildung 11-3a). Zwischen 1996 und 2002 ist der Anteil der mit ihrem Leben zufriedenen Menschen um circa sechs Prozentpunkte angestiegen. Seither ist dieser Anteil bemerkenswert stabil und hoch bei circa 78 Prozent geblieben. Dieser Wandel betrifft alle Altersgruppen gleichermaßen.

Abbildung 11-3: Anteile der Personen mit hoher Lebenszufriedenheit, nach Bildung und Landesteil, in den Jahren 1996, 2002, 2008 und 2014 (in Prozent)

a) Nach Erhebungsjahr

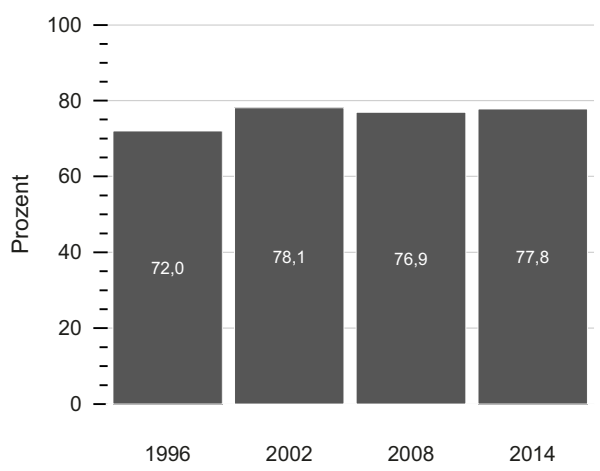


b) Nach Erhebungsjahr und Bildung

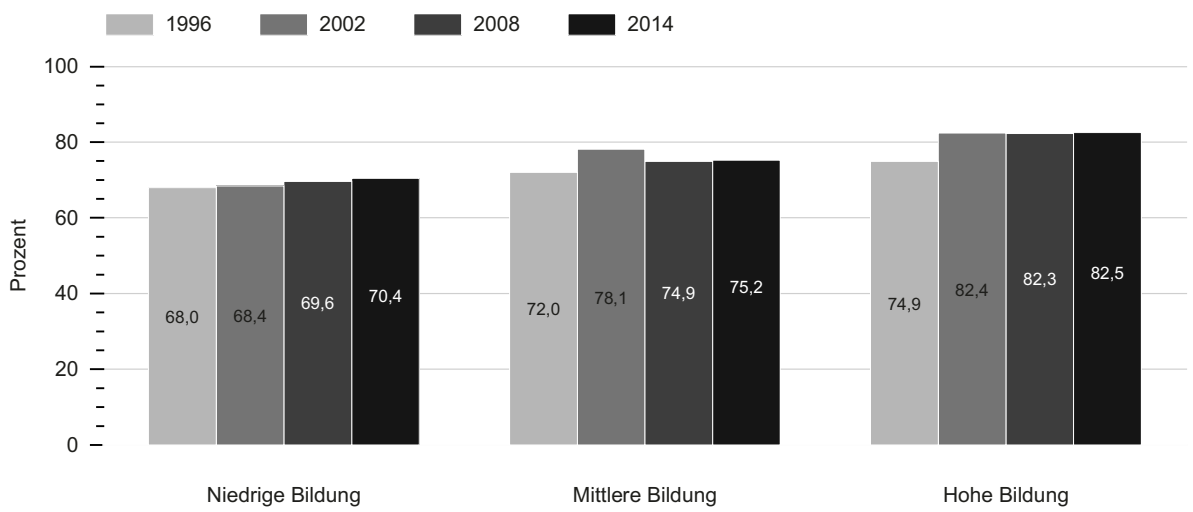

c) Nach Erhebungsjahr und Region

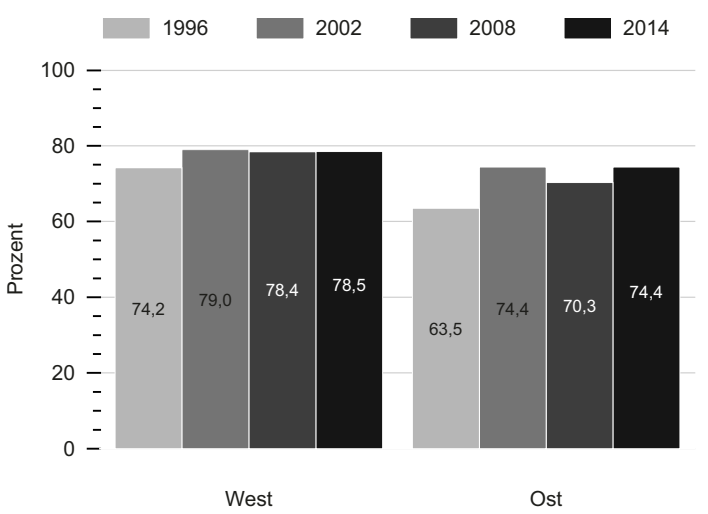

Quelle: DEAS $1996(n=4.004), 2002(n=2.775), 2008(n=4.396)$ und $2014(n=4.258)$, gewichtet, gerundete Angaben; $(p<, 05)$.

a) Signifikante Zunahme von 1996 zu 2002, Stabilität von 2002 bis 2014. b) Interaktion von Bildung und Erhebungsjahr signifikant. Signifikante Zunahme von 1996 zu 2002 und Stabilität von 2002 bis 2014 bei Personen mit mittlerer und hoher Bildung, keine signifikante Veränderung bei Niedriggebildeten. c) Interaktion von Region und Erhebungsjahr signifikant. Signifikant stärkere Zunahme von 1996 zu 2002 in Ostdeutschland als in Westdeutschland. Stabilität von 2002 zu 2014 in Westdeutschland. Für Ostdeutschland signifikante Unterschiede zwischen 2008 zu 2002 und zu 2014, kein signifikanter Unterschied zwischen 2002 und 2014.

Betrachtet man den Wandel getrennt nach Bildungsgruppen, hat sich von 1996 bis 2002 nur für Personen mit mittlerer und hoher Bildung eine Zunahme des Anteils an Personen mit hoher Lebenszufriedenheit gezeigt. Von 2002 bis 2014 sind die Werte in beiden Bildungsgruppen stabil geblieben (Abbildung 11-3b). Für Personen mit niedriger Bildung ist der Anteil an Personen mit hoher Lebenszufriedenheit dagegen über den gesamten Zeitraum stabil geblieben. Im Vergleich zu 1996 haben sich die Bildungsunterschiede also im Jahr 2014 verschärft. Der positive Wandel der Lebenszufriedenheit zeigt sich zudem stärker in Ostdeutschland, sodass die regionalen Unterschiede bei der Lebenszufriedenheit $2014 \mathrm{im}$ Vergleich zu den vorherigen Erhebungsjahren kleiner geworden sind (Abbildung 11-3c). 


\section{Der Wandel der depressiven Symptome unterscheidet sich zwischen den Altersgruppen - nur Ältere berichten 2014 weniger Symptome als 2002.}

Dagegen unterscheidet sich der Wandel hinsichtlich des Anteils von Personen mit zumindest leichten depressiven Symptomen nicht nach Bildungsgruppe oder Region; allerdings zeigt sich ein deutlicher Altersunterschied (Ab- bildung 11-4). In den jüngeren Altersgruppen, den 42- bis 65-Jährigen, ist der Anteil der Personen mit geringer depressiver Symptomatik zwischen 2002 und 2008 gesunken - danach jedoch wieder auf das Niveau von 2002 angestiegen. Gute Nachrichten gibt es dagegen von den älteren Altersgruppen, den 66-Jährigen und Älteren: Hier ist der Anteil der Personen mit zumindest geringer depressiver Symptomatik zwischen 2002 und 2008 gesunken und bis zum Jahr 2014 stabil geblieben.

Abbildung 11-4: Anteile der Personen mit mindestens leichten depressiven Symptomen, nach Alter, in den Jahren 2002, 2008 und 2014 (in Prozent)

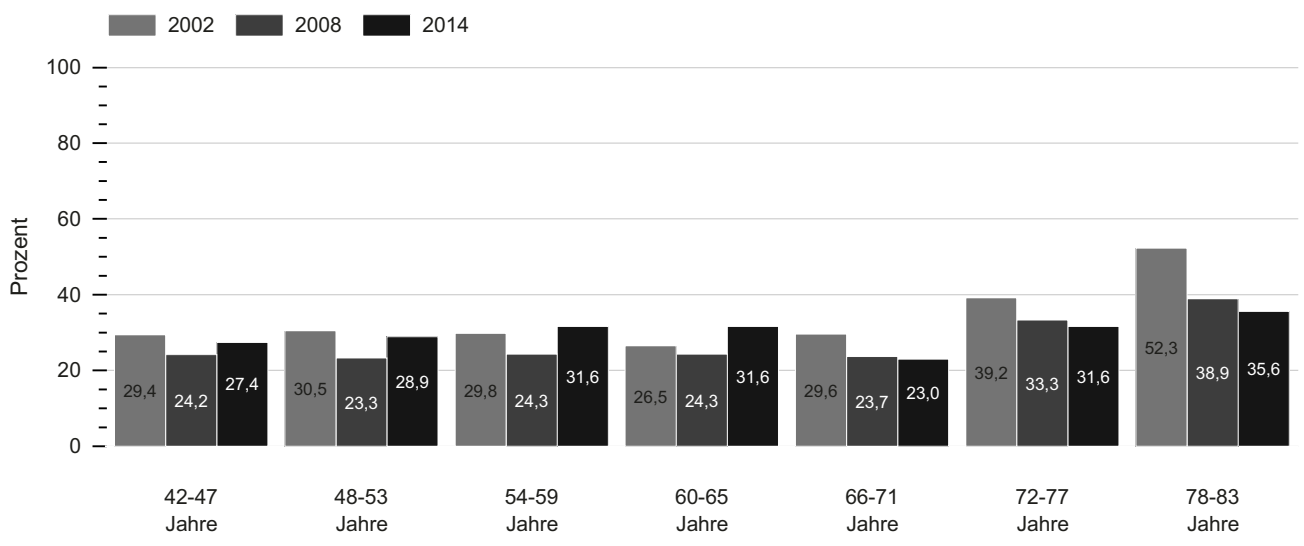

Quelle: DEAS $2002(n=2.873), 2008(n=6.072)$ und $2014(n=5.858)$, gewichtet, gerundete Angaben; $(p<, 05)$. In allen Altersgruppen signifikante Abnahme von 2002 zu 2008. Für die 42- bis 65-Jährigen signifikante Zunahme von 2008 zu 2014 und kein signifikanter Unterschied zwischen 2002 und 2014. Bei den über 65-Jährigen Stabilität von 2008 zu 2014.

\subsection{Diskussion und Implikationen}

Die Gesellschaft profitiert von einem hohen psychischem Wohlbefinden aller Bevölkerungsgruppen. Ein hohes Wohlbefinden ist eine Voraussetzung für gesellschaftliche Partizipation und Teilhabe der Bürgerinnen und Bürger. Ein Großteil der Menschen zwischen 40 und 85 Jahren berichtet auch 2014 von einer hohen Lebenszufriedenheit und einer geringen depressiven Symptomatik; nur eine Minderheit leidet unter klinisch auffälligen depressiven Symptomen. Aus der Forschung zum Zufriedenheitsparadox (Staudinger 2000) wissen wir, dass es im Alter durchaus möglich ist, trotz gesundheitlicher Einschränkungen eine hohe Lebenszufriedenheit $\mathrm{zu}$ bewahren. Die 70- bis 85 -Jährigen berichten sogar eine höhere Lebenszufriedenheit als die Personen im mittleren Erwachsenenalter. Zufriedenheit ist das Ergebnis von Wahrnehmungen, Beurteilungen und Entscheidungen: Wenn eine 
Person danach gefragt wird, ob sie mit ihrem Leben zufrieden ist, dann erfolgt die Antwort nach einer gedanklichen Prüfung der eigenen Lebenssituation, dem Vergleich mit Beurteilungsmaßstäben und der Entscheidung für ein Zufriedenheitsurteil. Damit handelt es sich bei der Lebenszufriedenheit um die kognitive Komponente des Wohlbefindens.

Im Unterschied dazu spiegelt die Frage nach depressiven Symptomen die Befindlichkeit einer Person wider. Dabei werden nicht allein die Gefühle einer Person erfragt, sondern auch ihre Motivation, ihre Gedanken sowie Probleme in der Beziehung mit anderen Menschen. Dies kann der Grund dafür sein, dass die Ergebnisse $\mathrm{zu}$ depressiven Symptomen weniger optimistisch ausfallen als zur Lebenszufriedenheit. Menschen zwischen 70 und 85 Jahren leiden häufiger unter zumindest leichten depressiven Symptomen als Jüngere, was stärkere Einschränkungen im Gesundheitszustand widerspiegeln könnte (vgl. Kapitel 8; Chang-Quan et al. 2010). Es gibt also für die 70- bis 85 -Jährigen eine Doppelbelastung von körperlichen und seelischen Beschwerden, die sich auch gegenseitig beeinflussen können.

Unterschiede bei der Lebenszufriedenheit und bei depressiven Symptomen spiegeln häufig eine Ungleichverteilung von Ressourcen wider. So berichten Frauen mehr depressive Symptome als Männer, was zum Beispiel an Ungleichheiten im Einkommen sowie in der beruflichen Situation oder an stärkeren Belastungen durch Vereinbarung von Erwerbstätigkeit mit Sorgetätigkeiten liegen könnte (vgl. Kapitel 12 und 15). Zudem haben diese Geschlechtsunterschiede über die Altersgruppen zugenommen und in der ältesten Altersgruppe berichten Frauen auch seltener eine hohe Lebenszufriedenheit als Männer. Dieser geschlechtsspezifische Altersgradient könnte in dem höheren Anteil verwitweter Frauen im Vergleich zu verwitweten Männern im hohen Alter begründet sein (Statistisches Bundesamt 2014). Der Verlust des Partners oder der Partnerin kann das Wohlbefinden in hohem Maße beeinträchtigen (Lucas, Clark, Georgellis, \& Diner 2003). Frauen stellen hier somit vor allem im hohen und sehr hohen Alter eine Risikogruppe dar. Besondere Aufmerksamkeit sollte zudem die Gruppe der Niedriggebildeten erfahren. Sowohl in der Lebenszufriedenheit als auch in den depressiven Symptomen zeigt sich eine deutliche Benachteiligung niedriger Bildungsgruppen, die in der Lebenszufriedenheit im Vergleich zu 1996 sogar zugenommen hat. Eine Ungleichverteilung von Ressourcen und Chancen kann also zu schlechteren Lebensbedingungen führen und so zu einem geringeren Wohlbefinden (vgl. Morgan, Robinson, \& Thompson 2015).

Von $2002 \mathrm{zu} 2008$ ist in allen Altersgruppen der Anteil an Personen mit zumindest leichten depressiven Symptomen zurückgegangen - bei den 42- bis 65-Jährigen steigt dieser Anteil jedoch im Jahr 2014 wieder auf das Niveau von 2002. Dagegen stabilisierte sich der niedrigere Anteil an Menschen mit zumindest leichten depressiven Symptomen bei den älteren Altersgruppen (66 bis 83 Jahre). Dies entspricht nicht der Befundlage zum Wandel hin zu mehr depressiven Symptomen, der in anderen Ländern $\mathrm{zu}$ beobachten ist (Brault et al. 2012; Keyes et al. 2014). Vielmehr zeigt sich im DEAS bei den älteren Altersgruppen eher eine positive Veränderung. Hier könnte der Wandel der depressiven Symptome mit dem Wandel der gesundheitlichen Einschränkungen von 2002 zu 2014 zusammenhängen (vgl. Kapitel 8). Es zeigt sich eine ähnliche Entwicklung: Für die über 65-Jährigen hat sich hier ein positiver Wandel gezeigt, während unter den 42- bis 65-Jährigen der Anteil an Personen mit Einschränkungen zugenommen hat. Auch die subjektive Gesundheit hat sich von $1996 \mathrm{zu} 2014$ bei den über 65-Jährigen verbessert (vgl. Kapitel 10). Eine gute medizinische Versorgung sollte demnach sowohl körperliche Erkrankungen und Einschränkungen als auch psychische Beschwerden in den Blick nehmen. Auch präventive Maßnahmen können dabei neben körperlichen Einschränkungen auch psychische Beschwerden thematisieren. Bewegungsprogramme können zum Beispiel nicht nur körperliche Erkrankungen und funktionale Einschränkungen verhindern oder abschwächen, sondern wirken sich auch positiv auf das Wohlbefinden aus.

Auch 25 Jahre nach der Wiedervereinigung zeigen sich noch Unterschiede zwischen Ost- 
und Westdeutschland, obwohl sich zumindest die Lebenszufriedenheit beider Landesteile seit 1996 weiter angeglichen hat. Dies entspricht auch den Befunden des SOEP (Priem \& Schupp 2014). Die Annäherung in der Lebenszufriedenheit reflektiert in vielen Bereichen die sich angleichenden Lebensbedingungen in Ost- und Westdeutschland. Trotz dieser Angleichung zeigen sich aber weiterhin deutliche Unterschiede zwischen beiden Landesteilen zum Beispiel in der materiellen Lage oder in der Einschätzung der Versorgung mit Ärztinnen und Ärzten (vgl. Kapitel 6 und 20). Ein Weg, um Unterschiede zwischen Ost- und Westdeutschland im Wohlbefinden weiter zu verringern, ist daher eine Angleichung der Lebensbedingungen. Neben den Lebensbedingungen sollten auch andere Bedingungen für Regionsunterschiede im psychischen Wohlbefinden, wie beispielsweise unterschiedliche Sozialisationserfahrungen und kulturelle Werthaltungen nicht außer Acht gelassen werden.

Zusammenfassend ist die Lebenszufriedenheit in der zweiten Lebenshälfte bei den allermeisten Menschen hoch. Anhaltende Nieder- geschlagenheit und depressive Verstimmungen sind nicht die Regel, leichte depressive Symptome sind aber im höheren Alter häufiger. Begreift man subjektives Wohlbefinden als ein Potenzial und eine Ressource zur gesellschaftlichen Teilhabe, so könnte dies über Beteiligungs- und Engagementangebote gesellschaftlich fruchtbar gemacht werden. Kann die Politik zu einem guten subjektiven Wohlbefinden - hoher Lebenszufriedenheit und geringer depressiver Symptomatik - überhaupt etwas beitragen? Welche Handlungsmöglichkeiten stehen der Politik offen? Die Voraussetzungen für ein hohes Wohlbefinden sind eine gute Gesundheit und eine gesicherte Lebenssituation, wozu nicht nur die private Ausgestaltung des Lebens sondern auch das Umfeld, die Infrastruktur, die medizinische Versorgung zählen - gesellschaftliche Bedingungen für ein gutes Leben im Alter. Dies gilt insbesondere für die Prävention, Erkennung und Behandlung depressiver Symptome im Alter. Es sind also nicht allein individuelle, sondern auch gesellschaftliche Maßnahmen erforderlich, um ein hohes Wohlbefinden in der zweiten Lebenshälfte aufrechtzuerhalten.

\section{Literatur}

Albani, C., Gunzelmann, T., Schmutzer, G., Grulke, N., Bailer, H., Blaser, G., Greyer, M., \& Brähler, E. (2005). Die emotionale Befindlichkeit älterer Menschen: Normierung des Profile of Mood States für über 60-Jährige. Zeitschrift für Gerontologie und Geriatrie, 38(6), 431-440. doi: 10.1007/s00391-005-0314-x.

Bjelland, I., Krokstad, S., Mykletun, A., Dahl, A. A., Tell, G. S., \& Tambs, K. (2008). Does a higher educational level protect against anxiety and depression? The HUNT study. Social Science \& Medicine, 66(6), 1334-1345. doi: 10.1016/j.socscimed.2007.12.019.

Brault, M.-C., Meuleman, B., \& Bracke, P. (2012). Depressive symptoms in the Belgian population: Disentangling age and cohort effects. Social Psychiatry and Psychiatric Epidemiology, 47(6), 903-915. doi: 10.1007/ s00127-011-0398-0.

Buber, I., \& Engelhardt, H. (2011). Der Zusammenhang zwischen Alter und depressiven Symptomen bei Männern und Frauen höheren Lebensalters in Europa. Erkenntnisse aus dem SHARE-Projekt. Zeitschrift für Bevölkerungswissenschaft, 36(1), 77-102. doi: 10.4232/10.CPoS-20.

Busch, M. A., Maske, U. E., Ryl, L., Schlack, R., \& Hapke, U. (2013). Prävalenz von depressiver Symptomatik und diagnostizierter Depression bei Erwachsenen in Deutschland: Ergebnisse der Studie zur Gesundheit Erwachsener in Deutschland. Bundesgesundheitsblatt, 56, 733-739. doi: 10.1007/s00103-013-1688-3.

Chang-Quan, H., Bi-Rong, D., Zhen-Chan, L., Ji-Rong, Y., \& Qing-Xiu, L. (2010). Chronic diseases and risk for depression in old age: A meta-analysis of published literature. Ageing Research Reviews, 9(2), 131-141. doi: 10.1016/j.arr.2009.05.005.

Fryers, T., Melzer, D., \& Jenkins, R. (2003). Social inequalities and the common mental disorders: A systematic review of the evidence. Social Psychiatry and Psychiatric Epidemiology, 38(5), 229-237. doi: 10.1007/ s00127-003-0627-2.

Gerstorf, D., Ram, N., Estabrook, R., Schupp, J., Wagner, G. G., \& Lindenberger, U. (2008). Life satisfaction 
shows terminal decline in old age: Longitudinal evidence from the German Socio-Economic Panel Study (SOEP). Developmental Psychology, 44(4), 1148-1159. doi: 10.1037/0012-1649.44.4.1148.

Glaesmer, H., Grande, G., Braehler, E., \& Roth, M. (2011). The German version of the Satisfaction with Life Scale (SWLS): Psychometric properties, validity, and population-based norms. European Journal of Psychological Assessment, 27(2), 127-132. doi: 10.1027/1015-5759/ a000058.

Hautzinger, M., \& Bailer, M. (1993). Allgemeine Depressions-Skala (ADS). Manual. Weinheim: Beltz.

Keyes, K. M., Nicholson, R., Kinley, J., Raposo, S., Stein, M. B., Goldner, E. M., \& Sareen, J. (2014). Age, period, and cohort effects in psychological distress in the United States and Canada. American Journal of Epidemiology, 179(10), 1216-1227. doi: 10.1093/aje/kwu029.

Laubach, W., Schumacher, J., Mundt, A., \& Brähler, E. (2000). Sozialschicht, Lebenszufriedenheit und Gesundheitseinschätzung. Ergebnisse einer repräsentativen Untersuchung der deutschen Bevölkerung. Sozial- und Präventivmedizin, 45(1), 2-12. doi: 10.1007/ bf01358994.

Lucas, R. E., Clark, A. E., Georgellis, Y., \& Diner, E. (2003). Reexamining adaptation and the set point model of happiness: Reactions to changes in marital status. Journal of Personality and Social Psychology, 84(3), 527-539. doi: 10.1037/0022-3514.84.3.527.

Morgan, J., Robinson, O., \& Thompson, T. (2015). Happiness and age in European adults: The moderating role of gross domestic product per capita. Psychology and Aging 30(3), 544-551. doi: 10.1037/pag0000034.

Müters, S., Hoebel, J., \& Lange, C. (2013). Diagnose Depression: Unterschiede bei Frauen und Männern. GBE kompakt, 4(2).

Pavot, W., \& Diener, E. (1993). Review of the Satisfaction with Life Scale. Psychological Assessment, 5(2), 164172. doi: 0.1037/1040-3590.5.2.164.

Pinquart, M., \& Sörensen, S. (2000). Influences of socioeconomic status, social network, and competence on subjective well-being in later life: A metaanalysis. Psychology and Aging, 15(2), 187-224. doi: 10.1037/0882-7974.15.2.187.

Priem, M., \& Schupp, J. (2014). Alle zufrieden: Lebensverhältnisse in Deutschland. DIW Wochenbericht, 81(40), 1001-1008.
Robert Koch-Institut (2014). Depression. Faktenblatt zu GEDA 2012: Ergebnisse der Studie "Gesundheit in Deutschland aktuell 2012«. Berlin: Robert Koch-Institut.

Rothermund, K., \& Brandtstädter, J. (2003). Depression in later life: Cross-sequential patterns and possible determinants. Psychology and Aging, 18(1), 80. doi: 10.1037/0882-7974.18.1.80

Schienle, A. (2007). Geschlechterdifferenzen in der Emotionalität aus der Sicht des Neuroimaging. In: S. Lautenbacher, O. Güntürkün \& M. Hausmann (Hrsg.) Gehirn und Geschlecht - Neurowissenschaft des kleinen Unterschieds zwischen Mann und Frau (S. 143-159). Heidelberg: Springer.

Schöllgen, I., Huxhold, O., \& Tesch-Römer, C. (2010). Socioeconomic status and health in the second half of life: Findings from the German Ageing Survey. European Journal of Ageing, 7(1), 17-28. doi: 10.1007/ s10433-010-0140-x.

Schupp, J., Goebel, J., Kroh, M., \& Wagner, G. G. (2013). Zufriedenheit in Deutschland so hoch wie nie nach der Wiedervereinigung: Ostdeutsche signifikant unzufriedener als Westdeutsche. DIW Wochenbericht, 80(47), 34-43.

Statistisches Bundesamt (2014). Haushalte und Familien - Ergebnisse des Mikrozensus: Bevölkerung und Erwerbstätigkeit. Wiesbaden: Statistisches Bundesamt.

Staudinger, U. M. (2000). Viele Gründe sprechen dagegen, und trotzdem geht es vielen Menschen gut: Das Paradox des subjektiven Wohlbefindens. Psychologische Rundschau, 51(4), 185-197. doi: 10.1026//00333042.51.4.185.

Tesch-Römer, C., Motel-Klingebiel, A., \& Tomasik, M. J. (2008). Gender differences in subjective well-being: Comparing societies with respect to gender equality. Social Indicator Research, 85(2), 329-349. doi: 10.1007/s11205-007-9133-3.

Tesch-Römer, C., Wiest, M., \& Wurm, S. (2010). Subjektives Wohlbefinden. In: A. Motel-Klingebiel, S. Wurm \& C. Tesch-Römer (Hrsg.) Altern im Wandel: Befunde des Deutschen Alterssurveys (DEAS) (S. 263-283). Stuttgart: Kohlhammer.

Veenhoven, R. (2008). Sociological theories of subjective well-being. In: M. Eid \& R. J. Larsen (Hrsg.) Subjective Well-being. New York: Guilford.

Open Access Dieses Kapitel wird unter der Creative Commons Namensnennung 2.5 International Lizenz (http://creativecommons.org/licenses/by/2.5/deed.de) veröffentlicht, welche die Nutzung, Vervielfältigung, Bearbeitung, Verbreitung und Wiedergabe in jeglichem Medium und Format erlaubt, sofern Sie den/die ursprünglichen Autor(en) und die Quelle ordnungsgemäß nennen, einen Link zur Creative Commons Lizenz beifügen und angeben, ob Änderungen vorgenommen wurden.

Die in diesem Kapitel enthaltenen Bilder und sonstiges Drittmaterial unterliegen ebenfalls der genannten Creative Commons Lizenz, sofern sich aus der Abbildungslegende nichts anderes ergibt. Sofern das betreffende Material nicht unter der genannten Creative Commons Lizenz steht und die betreffende Handlung nicht nach gesetzlichen Vorschriften erlaubt ist, ist für die oben aufgeführten Weiterverwendungen des Materials die Einwilligung des jeweiligen Rechteinhabers einzuholen. 